\begin{tabular}{|l|}
\hline A\&A manuscript no. \\
(will be inserted by hand later) \\
\hline Your thesaurus codes are: \\
$\mathbf{1 3 . 1 8 . 1}-\mathbf{0 2 . 1 8 . 5}$ \\
\hline
\end{tabular}

ASTRONOMY AND

ASTROPHYSICS

6.9 .2018

\title{
Synchrotron Spectra and Ages of Compact Steep Spectrum Radio Sources
}

\author{
M. Murgia ${ }^{1,2}$, C. Fanti ${ }^{1,3}$, R. Fanti ${ }^{1,3}$, L. Gregorini ${ }^{1,3}$, U. Klein ${ }^{4}$, K.-H. Mack ${ }^{1,4}$, and M. Vigotti ${ }^{1}$ \\ 1 Istituto di Radioastronomia del CNR, Via Gobetti 101, I-40121 Bologna, Italy \\ 2 Dipartimento di Astronomia, Università di Bologna, Via Zamboni 33, I-40126 Bologna, Italy \\ 3 Dipartimento di Fisica, Università di Bologna, Via Irnerio 46, I-40126 Bologna, Italy \\ 4 Radioastronomisches Institut der Universität Bonn, Auf dem Hügel 71, D-53121 Bonn, Germany
}

Received, accepted

\begin{abstract}
The high-frequency integrated spectra of Compact Steep Spectrum (CSS) sources show breaks with a moderate spectral steepening well fitted by continuous injection synchrotron spectra. In lobe-dominated CSS sources the radiative ages deduced by the synchrotron theory are in the range of up to $10^{5}$ years, if equipartition magnetic fields are assumed. These radiative ages are well correlated with the source size indicating that the CSS sources are young. In order to maintain the frustration scenario, in which the sources' lifetimes are $\approx 10^{7}$ years, their equipartition magnetic field would be systematically decreased by a factor $\gtrsim 20$. To complete the sample used in this work, we conducted observations at $230 \mathrm{GHz}$ with the IRAM 30-m telescope of those sources which did not have such high-frequency observations up to now.
\end{abstract}

Key words: Radio continuum: galaxies; radiation mechanisms: non-thermal

\section{Introduction}

Flux density limited radio source samples are known to contain a large portion of compact sources with high frequency spectral index $\alpha \geq 0.5\left(\mathrm{~S}_{\nu} \sim \nu^{-\alpha}\right)$ and angular sizes below $2^{\prime \prime}$. The corresponding projected linear sizes are typically $\leq 15 \mathrm{kpd}$. Fanti et al. (1990) have shown that the majority of these CSS sources cannot be larger sources foreshortened by projection effects, which means that their radio emission originates on sub-galactic scales.

It is obvious that one should investigate the connection between CSS and larger radio sources. Two scenarios have been proposed that would naturally explain the observed small sizes. First, they could reflect an early stage

Send offprint requests to: M. Murgia

1 We assume $\mathrm{H}_{0}=100 \mathrm{~km} \mathrm{~s}^{-1} \mathrm{Mpc}^{-1}$ and $\mathrm{q}_{0}=0.5$ in the evolution of radio sources. This is the youth scenario (Phillips \& Mutel 1982; Carvahlo 1985). The second possibility is that the unusual conditions in the interstellar medium of their host galaxies, such as a higher density and/or the presence of turbulence, inhibit the radio source from growing to larger sizes. This is the frustration scenario (van Breugel et al. 1984).

Most CSS sources exhibit double-lobed structures such as seen in classical radio galaxies. This feature is common to both quasars and galaxies. Quite a few of these are symmetric, which gave rise to the terms CSO (compact symmetric objects, with sizes $\leq 0.5 \mathrm{kpc}$ ) and MSO (medium-size symmetric objects, with sizes $>0.5 \mathrm{kpc}$ ). These sources are considered as scaled-down versions of larger-sized double radio sources (Fanti et al. 1995). A minority of CSS sources is made up by sources with complex or highly asymmetric structures. These are mostly quasars, with most of their luminosity provided by the jet. The distortions may be caused by jet bending, which is further amplified by strong projection.

A plausible organization scheme for these different morphological classes is to identify CSO and MSO with progenitors of large doubles ('baby radio galaxies'), while the asymmetric CSS sources could represent frustrated radio sources.

\section{Sample selection}

The sample we choose for this study consists of:

1) the sample selected from the $3 \mathrm{CR}$ and the Peacock \& Wall (1981; PW) catalogues (Fanti et al. 1995), and 2) nine sources extracted from the B3 VLA sample (Vigotti et al. 1989).

1) The $3 \mathrm{CR} / \mathrm{PW}$ sample (38 sources) was established on the basis of the well-known criteria: linear size $<15$ kpc, spectral index $\alpha>0.5$ and a limit in radio power $\mathrm{P} \geq 10^{26.8} \mathrm{~W} / \mathrm{Hz}$ at $178 \mathrm{MHz}$ for the $3 \mathrm{CR}$, or $\mathrm{P} \geq 10^{26}$ $\mathrm{W} / \mathrm{Hz}$ at $2.7 \mathrm{GHz}$ for the $\mathrm{PW}$. High resolution structure 
information is available for these sources from the literature. In the framework of this project we observed at $230 \mathrm{GHz}$ the 17 sources for which the flux densities at this frequency were not available from literature.

2 ) The second complete sample of CSS sources was selected from the "strong" section of the B3 VLA catalogue $\left(S_{408 \mathrm{MHz}} \geq 0.8 \mathrm{Jy}\right)$ according to the following definitions: $\alpha_{408 \mathrm{MHz}}^{1.4 \mathrm{GHz}}>0.5$ up to $10 \mathrm{GHz}$, and a size of $\lesssim 15 \mathrm{kpc}$. Sources with $\alpha_{408 \mathrm{MHz}}^{1.4 \mathrm{GHz}}<0.5$ have also been included if their spectrum is steeper than $\alpha=0.5$ at $\nu>1.4 \mathrm{GHz}$. These are Giga-Hertz-peaked spectrum (GPS) candidate sources. This sample contains 83 objects. The sample has been recently observed with the VLA at 5 and $8.4 \mathrm{GHz}$, with resolutions of $\sim 00^{\prime \prime} 2$. Additional observations with VLBI arrays are planned. Only about $60 \%$ of these sources have at the moment either a photometric or a spectroscopic redshift, therefore we limited the list to the objects which could be observed at $230 \mathrm{GHz}$ to the latter. Due to scheduling constraints we only observed 9 sources of the B3 VLA sample.

\section{Observations and data reduction}

The observations were done with the IRAM 30-m telescope near Granada, Spain between 10th Feb. and 15th Feb., 1998. The receiver we used was the 37-channel bolometer described by Kreysa et al. (1998), with feeds arranged in a hexagon around the central feed, and beam separations of $20^{\prime \prime}$. The sensitivity of each channel was $70 \mathrm{mJy} / \mathrm{s}$. The beam size was $10^{\prime \prime} 5$ as derived from pointing scans. All our sources were point-like to this beam. Opacity measurements were made via skydips, from which we derived zenith opacities of 0.1-0.8. Despite the high atmospheric attenuation of the signal, the observations (generally done at high source elevations) could be performed satisfactorily, owing to the relatively quiet atmosphere present during our observing run (resulting in a relatively low sky noise). The calibration factor to convert the observed counts into flux densities was determined by observing the source K3-50A, which has a total flux density of $10 \mathrm{Jy}$ and a peak flux density of $7.6 \mathrm{Jy}$ for the beam size of our measurements. The measures were finally corrected for atmospheric attenuation and the elevationdependent gain of the telescope. The pointing was frequently checked by cross-scanning the sources $0923+392$, $1144+402,1308+326$, and $1418+546$. The pointing accuracy was found to be $\sim 3^{\prime \prime}$. The same sources were used to adjust the focus at regular intervals.

The sources were observed in an ON-OFF mode in which the wobbling secondary mirror of the telescope moved at a frequency of $2 \mathrm{~Hz}$ between the source position and positions located at $\pm 60^{\prime \prime}$ in azimuth. Each source was observed between 3 and 5 times, with $10 \mathrm{ON}$ OFF pairs each. The differences of the flux densities between the main and the reference beams were averaged by weighting inversely proportional to the square of the r.m.s. noise of each measurement. The resulting integration time spent on each source was thus typically 50 minutes, and the final r.m.s. noise of each measurement was between 2 and $5 \mathrm{mJy}$. In addition, the calibration may be uncertain by $15 \%$ owing to day-to-day variations in the system gain.

The data were recorded with all receivers, with the central one pointing at the source. As the angular extents of the target sources are in the range of a few arcseconds, the six outer channels contain only sky emission. Hence, they were used to subtract a mean sky level from the central channel. Using this procedure, we assumed that the scale size of the fluctuations of the sky emission is large compared to the total coverage of the bolometer array $\left(60^{\prime \prime}\right)$, which corresponds to $1.5 \mathrm{~m}$ at a distance of $5 \mathrm{~km}$. Together with the wobbling rate, this ensures efficient rejection of sky noise, since the time scale of atmospheric fluctuations is much larger (see e.g. Altenhoff et al. 1987).

In Tab. 1 we list the measured flux densities and 1- $\sigma$ errors, inclusive of noise and calibration uncertainty, at $230 \mathrm{GHz}$.

Table 1. Flux densities at $230 \mathrm{GHz}$

\begin{tabular}{|c|c|c|c|}
\hline \multirow[t]{2}{*}{ Name } & \multicolumn{2}{|c|}{ Pointing centre (B1950) } & \multirow{2}{*}{$\begin{array}{c}\mathrm{S}_{230} \\
{[\mathrm{mJy}]}\end{array}$} \\
\hline & $\alpha$ & $\delta$ & \\
\hline \multicolumn{4}{|l|}{ 3C-PW } \\
\hline $0404+76$ & 040400.13 & 764852.50 & $115 \pm 18$ \\
\hline $0428+20$ & 042806.86 & 203109.13 & $62 \pm 11$ \\
\hline $0740+383 \mathrm{C} 186$ & 074056.77 & 380030.97 & $5 \pm 4$ \\
\hline $0758+143 \mathrm{C} 190$ & 075845.05 & 142304.40 & $8 \pm 3$ \\
\hline $1005+073 \mathrm{C} 237$ & 100522.04 & 074458.56 & $14 \pm 5$ \\
\hline $1019+223 \mathrm{C} 241$ & 101909.38 & 221439.63 & $2 \pm 3$ \\
\hline $1153+31$ & 115344.08 & 314416.00 & $9 \pm 3$ \\
\hline $1203+643 \mathrm{C} 268.3$ & 120354.08 & 643018.40 & $1 \pm 2$ \\
\hline $1225+36$ & 122530.77 & 365147.00 & $0 \pm 3$ \\
\hline $1358+62$ & 135858.36 & $\begin{array}{lll}62 & 25 & 06.71\end{array}$ & $29 \pm 6$ \\
\hline $1416+063 \mathrm{C} 298$ & 141638.80 & 064221.30 & $8 \pm 4$ \\
\hline $1447+773 \mathrm{C} 305.1$ & 144749.35 & 770846.65 & $2 \pm 3$ \\
\hline $1517+203 \mathrm{C} 318$ & 151750.63 & 202652.95 & $5 \pm 3$ \\
\hline $1634+623 \mathrm{C} 343$ & 163401.06 & 625141.80 & $12 \pm 4$ \\
\hline $1637+623 \mathrm{C} 343.1$ & 163755.29 & 624034.24 & $7 \pm 4$ \\
\hline $1819+39$ & 181942.33 & 394115.00 & $5 \pm 4$ \\
\hline $1829+29$ & 182917.93 & 290458.29 & $9 \pm 4$ \\
\hline \multicolumn{4}{|l|}{ B3 VLA } \\
\hline $0809+404$ & 080931.62 & 402802.76 & $11 \pm 3$ \\
\hline $0810+460 \mathrm{~B}$ & 081058.61 & 460548.12 & $2 \pm 2$ \\
\hline $1025+390 \mathrm{~B}$ & 102549.34 & 385957.55 & $46 \pm 8$ \\
\hline $1128+455$ & 112856.40 & 453124.57 & $4 \pm 3$ \\
\hline $1159+395$ & 115916.35 & 393552.89 & $5 \pm 3$ \\
\hline $1225+442$ & 122515.70 & 441717.23 & $1 \pm 3$ \\
\hline $1233+418$ & 123310.88 & 415338.00 & $11 \pm 4$ \\
\hline $1242+410$ & 124226.39 & 410429.97 & $83 \pm 13$ \\
\hline $1350+432$ & 135024.04 & 431409.40 & $4 \pm 3$ \\
\hline
\end{tabular}



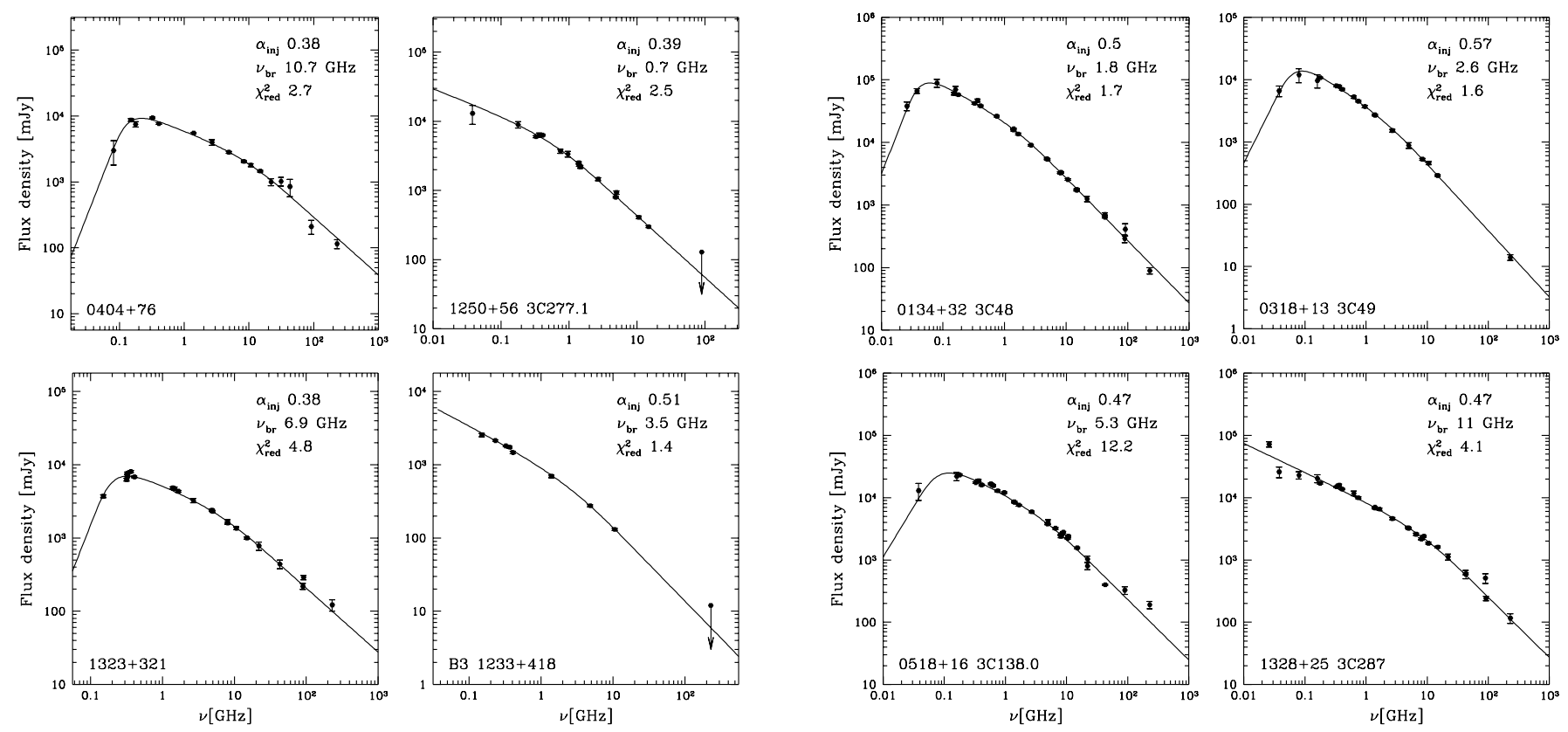

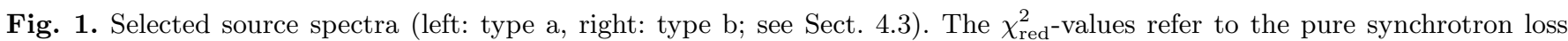
models. The self-absorbed part of the spectrum has been modelled in a subsequent fit procedure.

\section{Results and discussion}

For each source we have compiled flux densities at different frequencies from the literature, mostly from Kühr et al. (1979) and from the CATS database (Verkhodanov et al. 1997). Our new measures at $230 \mathrm{GHz}$ have been added to the compilation. All flux densities have been brought to the BGPW scale (Baars et al. 1977). Examples of source spectra are shown in Fig. 1. For the fit algorithm described in Sect. 4.2 we have assumed flux densities less than $3 \sigma$ to be upper limits only.

Most of the sources show significant departure from the classical power law which describes a zero age transparent synchrotron spectrum from a relativistic electron population with power law energy distribution. The deviations from the power law are of the following type: a) a lowfrequency turnover (the most conspicuous deviation); b) a steepening at high frequencies. High-frequency flattening, if any, is quite rare. The above deviations are interpreted as due to synchrotron self-absorption and to particle energy losses, respectively. In order to describe them, we have fitted the compiled flux densities with a synchrotron aged spectrum $\mathrm{S}_{\text {aged }}(\nu)$ (described in the next section), modified by low-frequency absorption, as follows (Pacholczyk 1970):

$\mathrm{S}(\nu) \propto\left(\nu / \nu_{1}\right)^{\alpha+\beta}\left(1-e^{-\left(\nu / \nu_{1}\right)^{-(\alpha+\beta)}}\right) \cdot \mathrm{S}_{\text {aged }}(\nu)$

where $\nu_{1}$ is the frequency at which the optical depth is equal to 1 . In case of an homogeneous synchrotron selfabsorbed source $\beta=2.5$, while $\alpha$ is the not aged spectral index in the transparent frequency range.

\subsection{The synchrotron aged spectrum model}

We assume that the radio source evolution is described by a continuous injection model, where the sources are continuously replenished by a constant flow of fresh relativistic particles with a power law energy distribution, with exponent $\delta$. It is well known that, under these assumptions, the radio spectrum has a standard shape (Kardashev 1962), with spectral index $\alpha_{\mathrm{inj}}=(\delta-1) / 2$ below a critical frequency $\nu_{\mathrm{br}}$ and $\alpha_{\mathrm{h}}=\alpha_{\mathrm{inj}}+0.5$ above $\nu_{\mathrm{br}}$. If there is no expansion and the magnetic field is constant, the frequency $\nu_{\mathrm{br}}$ (in $\mathrm{GHz}$ ) depends on the elapsed time since the source formation, $\tau_{\text {syn }}$ (in Myrs), the intensity of the magnetic field $B$ (in $\mu \mathrm{G}$ ) and the magnetic field equivalent to the microwave background $B_{\mathrm{CMB}}=3.25(1+z)^{2}$ (in $\mu \mathrm{G}$ ) as:

$\tau_{\mathrm{syn}}=1610 \frac{B^{0.5}}{B^{2}+B_{\mathrm{CMB}}^{2}} \frac{1}{\left[\nu_{\mathrm{br}}(1+z)\right]^{1 / 2}}$

The whole spectral shape cannot be described by an analytic equation, the two behaviours described being only the asymptotical ones, and has to be computed numerically. This model is referred as continuous injection (CI). Fitting the spectral data to the numerically computed CI spectrum, one obtains the break frequency $\nu_{\mathrm{br}}$, from which the source age is obtained if the magnetic field is known. This simple model does not consider expansion effects, which may be important if the source is young. So, the simplest variant of the original model is the one in which the radiating particles loose energy through expansion and the magnetic field changes according to flux conservation (Kardashev 1962, CIE). An alternative possibility to be 
considered, in an expanding source, is that the magnetic field changes less rapidly than in the flux conserving assumption because of a continuous magnetic flux input associated to the fresh particles injection. We set $B \propto t^{-\mathrm{m}}$, where for $\mathrm{m}=2$ we get the flux conserving expansion. This is referred as CIm model. In our special case we assume $\mathrm{m}=1$, consistent with the models applied by Baldwin (1982) or Begelman (1996). Although the theoretical background to these models is in the Kardashev paper, we have decided to present in the Appendix the detailed development. The spectral shapes of the CIE and CIm models have been computed again numerically. The break frequency for the CIE model is sixteen times higher than in the CI case, for equal elapsed time and final magnetic field intensity (Kardashev 1962). In the CIm model, instead, one finds that the break frequency is $(2.5 \mathrm{~m}-1)^{2}$ larger than in the CI model. The asymptotic behaviours at frequencies lower and larger than the break frequency are the same as in the CI model. However the steepening occurs over a broader frequency interval. In order to better emphasize the differences between these three models it is useful to consider, together with the usual flux-frequency representation, the point-to-point spectral index defined as

$\alpha\left(\nu / \nu_{\mathrm{br}}\right)=-\frac{d \log \mathrm{S}_{\mathrm{aged}}\left(\nu / \nu_{\mathrm{br}}\right)}{d \log \nu / \nu_{\mathrm{br}}}$

Both representations are shown in Fig. 2. While in the flux-frequency plane the differences are hardly visible, they can be much better traced in the point-to-point spectral index behaviour. Note that the displayed figures only show the theoretical differences. In practice the observed spectra permit only fits in the flux-frequency plane.

\subsection{The spectral fits}

Spectral fits to the spectra have been made with the CI, CIE, and CIm models. They allow us to determine the non-aged spectral index $\alpha_{\mathrm{inj}}$ and the break frequency $\nu_{\mathrm{br}}$, that, together with the normalization, are the three free parameters characterizing all the models. The best fits are, surprisingly, obtained with the CI model. The other models have steepenings which are too gradual for the majority of the spectra. An example is given in Fig. 3. The reduced $\chi_{\text {red }}^{2}$ of the models including adiabatic expansion and a variable magnetic field is always greater (typically twice) than that of the CI model (Fig. 4). The CI fits appear quite good, even in cases of high $\chi_{\text {red }}^{2}$ values, which, at visual inspection, appear more due to an under-estimation of the flux density errors than to a poor fit of the spectral model on the data.

The majority of the spectra show a clear break frequency, with a change of slope $\Delta \alpha \sim 0.5$. We stress that there is no evidence for spectral steepening with $\Delta \alpha$ significantly larger than 0.5 . Only a few sources are fitted by simple power laws. In these cases $\nu_{\mathrm{br}}$ could be either very

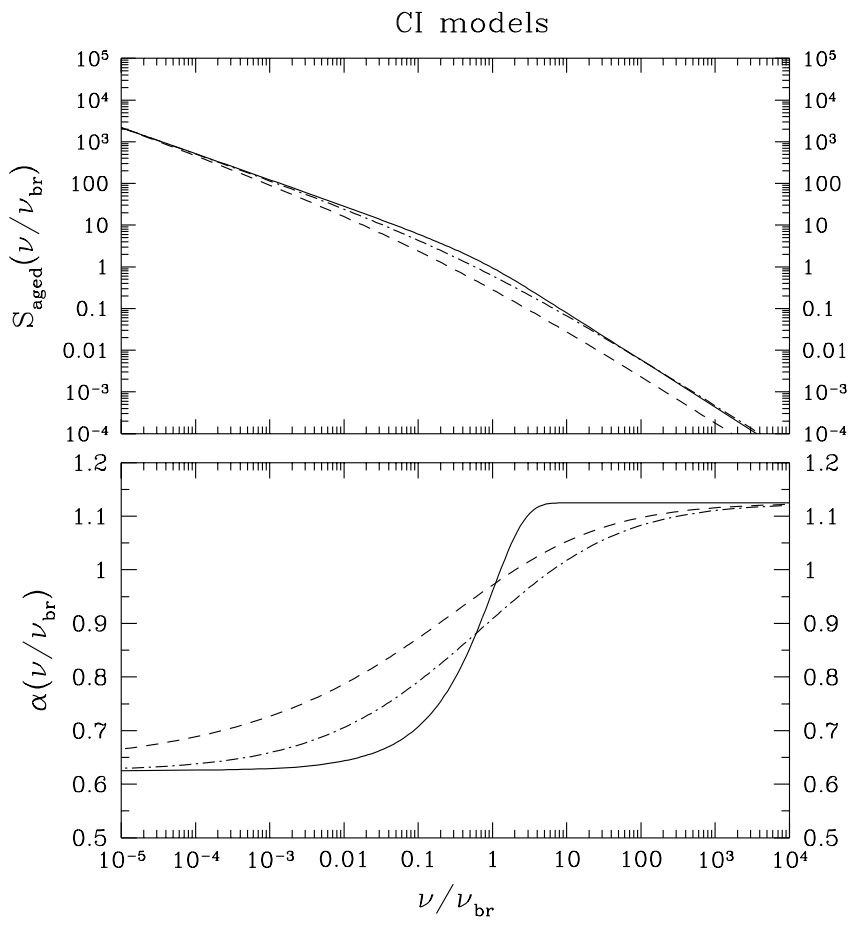

Fig. 2. The three continuous injection models described in the text. The solid line corresponds to the CI, the dashed line to the CIE and the dot-dashed line to the CIm. On the top the flux density (arbitrary units) is plotted as a function of the ratio $\nu / \nu_{\text {br }}$ (flux-frequency plane), on the bottom the point-to-point spectral index is shown as a function of $\nu / \nu_{\mathrm{br}}$. The injection spectral index is the same for all three models $\alpha_{\mathrm{inj}}=0.625$.

high ( $\gtrsim 100 \mathrm{GHz}$ ) or very low ( $\lesssim$ few $100 \mathrm{MHz}$ ). In these sources we have preferred the low frequency choice, since for the high frequency one would have implied abnormally high values for $\alpha_{\text {inj }}$ as compared to the other sources.

We also note that only a very small fraction of the CSS sources, if any, shows some evidence of flux density excess at high frequency, as it would be caused by a flat spectrum core or by thermal dust emission. Perhaps the only case is $3 \mathrm{C} 138$, where the core, known from VLBI observations, shows up in the integrated spectrum at $230 \mathrm{GHz}$ only.

It may appear surprising that the fits with the assumed spectral model are so good. In fact the sources of the sample consist of several components, as lobes and jets and hot spots, where physical conditions can differ from one another and therefore also break frequencies may be different. It is likely that the spectrum is dominated by the brighter component(s). In addition, one could imagine that some confusion might have occurred between genuine spectral steepening due to energy losses and the lowfrequency turnover due to absorption processes. We feel that this is a minor problem, but it is difficult to quantify it (see, however, next section).

The break frequencies range from a few hundred $\mathrm{MHz}$ to tens of $\mathrm{GHz}$. At low frequencies, as said above, the 
Table 2. Physical Parameters

\begin{tabular}{|c|c|c|c|c|c|c|c|c|}
\hline Source & redshift & $\begin{array}{c}\mathrm{LS} \\
{[\mathrm{kpc}]}\end{array}$ & $\begin{array}{c}\nu_{\mathrm{br}} \\
{[\mathrm{GHz}]}\end{array}$ & $\alpha_{\text {inj }}$ & $\begin{array}{c}B_{\mathrm{eq}} \\
{\left[10^{-3} \mathrm{G}\right]}\end{array}$ & $\begin{array}{c}\tau_{\text {syn }} \\
{\left[10^{3} \mathrm{yrs}\right]}\end{array}$ & $v_{\exp } / \mathrm{c}$ & type \\
\hline \multicolumn{9}{|l|}{ 3C-PW } \\
\hline $0127+23(3 \mathrm{C} 43)$ & 1.46 & 9.40 & $>100$ & 0.76 & 5.0 & 0.3 & 55.0 & $\mathrm{~b}$ \\
\hline $0134+32(3 \mathrm{C} 48)$ & 0.37 & 2.10 & 1.8 & 0.50 & 2.0 & 11.3 & 0.60 & $\mathrm{~b}$ \\
\hline $0138+13(3 \mathrm{C} 49)$ & 0.62 & 3.60 & 2.6 & 0.57 & 7.0 & 1.3 & 4.39 & b \\
\hline $0221+67(3 \mathrm{C} 67)$ & 0.31 & 6.80 & 2.2 & 0.56 & 0.7 & 51.0 & 0.22 & a \\
\hline $0223+34$ & 1.00 & 3.80 & 24.1 & 0.38 & 9.4 & 0.3 & 24.20 & $\mathrm{~b}$ \\
\hline $0316+16$ & 1.00 & 1.20 & 12.0 & 0.81 & 9.8 & 0.3 & 5.74 & $\mathrm{~b}$ \\
\hline $0345+33(3 \mathrm{C} 93.1)$ & 0.24 & 1.20 & 1.6 & 0.58 & 1.0 & 35.8 & 0.05 & $?$ \\
\hline $0404+76$ & 0.60 & 0.53 & 10.7 & 0.38 & 4.9 & 1.1 & 0.76 & $\mathrm{a}$ \\
\hline $0428+20$ & 0.22 & 0.45 & 7.2 & 0.38 & 5.8 & 1.2 & 0.59 & b \\
\hline $0429+41(3 \mathrm{C} 119)$ & 1.02 & 0.75 & 9.5 & 0.49 & 8.0 & 0.5 & 2.36 & $\mathrm{~b}$ \\
\hline $0518+16(3 \mathrm{C} 138)$ & 0.76 & 2.90 & 5.3 & 0.47 & 1.0 & 16.7 & 0.28 & $\mathrm{~b}$ \\
\hline $0538+49(3 \mathrm{C} 147)$ & 0.55 & 2.40 & 1.6 & 0.44 & 2.5 & 8.3 & 0.47 & $\mathrm{~b}$ \\
\hline $0740+38(3 \mathrm{C} 186)$ & 1.06 & 8.20 & 0.3 & 0.75 & 0.7 & 113.2 & 0.12 & $\mathrm{a}$ \\
\hline 0758+14 (3C190) & 1.20 & 14.10 & 30.3 & 0.79 & 0.7 & 10.6 & 2.15 & $\mathrm{~b}$ \\
\hline $1005+07(3 \mathrm{C} 237)$ & 0.88 & 4.50 & 2.4 & 0.59 & 1.2 & 18.1 & 0.40 & $\mathrm{a}$ \\
\hline $1019+22(3 \mathrm{C} 241)$ & 1.62 & 2.80 & 1.1 & 0.77 & 2.2 & 9.3 & 0.49 & $\mathrm{a}$ \\
\hline $1153+31$ & 1.56 & 3.20 & 6.9 & 0.69 & 1.7 & 5.4 & 0.95 & $\mathrm{a}$ \\
\hline $1203+64(3 \mathrm{C} 268.3)$ & 0.37 & 3.90 & 4.4 & 0.66 & 0.7 & 35.4 & 0.18 & $\mathrm{a}$ \\
\hline $1225+36$ & 1.98 & 0.17 & 3.9 & 0.64 & 18.0 & 0.2 & 1.41 & $\mathrm{~b}$ \\
\hline $1250+56(3 \mathrm{C} 277.1)$ & 0.32 & 4.40 & 0.7 & 0.39 & 0.4 & 204.3 & 0.04 & $\mathrm{a}$ \\
\hline $1323+32$ & 0.37 & 0.18 & 6.9 & 0.38 & 5.1 & 1.4 & 0.20 & $\mathrm{a}$ \\
\hline $1328+30(3 \mathrm{C} 286)$ & 0.85 & 14.20 & 7.3 & 0.38 & 13.0 & 0.3 & 77.82 & $\mathrm{~b}$ \\
\hline $1328+25(3 \mathrm{C} 287)$ & 1.06 & 0.40 & 10.7 & 0.47 & 3.5 & 1.7 & 0.39 & $\mathrm{~b}$ \\
\hline $1358+62$ & 0.43 & 0.16 & 71.9 & 0.70 & 9.0 & 0.2 & 1.39 & $\mathrm{~b}$ \\
\hline $1416+06(3 \mathrm{C} 298)$ & 1.44 & 9.10 & $<0.1$ & 0.50 & 1.6 & $>50$ & $<0.3$ & $\mathrm{a}$ \\
\hline $1443+77(3 \mathrm{C} 303.1)$ & 0.27 & 5.00 & 0.8 & 0.64 & 0.6 & 110.2 & 0.07 & $\mathrm{a}$ \\
\hline $1447+77$ (3C305.1) & 1.13 & 9.00 & 0.8 & 0.62 & 0.4 & 151.3 & 0.10 & a \\
\hline $1458+71(3 \mathrm{C} 309.1)$ & 0.90 & 7.80 & 109.4 & 0.65 & 7.0 & 0.6 & 21.0 & $\mathrm{~b}$ \\
\hline $1517+20(3 \mathrm{C} 318)$ & 0.75 & 7.80 & 4.8 & 0.69 & 1.5 & 9.6 & 1.32 & $\mathrm{~b}$ \\
\hline $1607+26$ & 0.47 & 0.18 & 6.3 & 0.71 & 10.0 & 0.5 & 0.55 & $\mathrm{a}$ \\
\hline $1634+62(3 \mathrm{C} 343)$ & 0.99 & 0.70 & 3.8 & 0.65 & 2.0 & 6.5 & 0.17 & $?$ \\
\hline $1637+62(3 \mathrm{C} 343.1)$ & 0.75 & 1.30 & 1.7 & 0.62 & 1.5 & 16.0 & 0.13 & $\mathrm{a}$ \\
\hline $1819+39$ & 0.40 & 3.10 & 3.3 & 0.73 & 6.0 & 1.6 & 3.13 & $\mathrm{~b}$ \\
\hline $1829+29$ & 0.60 & 9.30 & 12.1 & 0.63 & 6.0 & 0.8 & 19.12 & $\mathrm{~b}$ \\
\hline $2248+71(3 \mathrm{C} 454.1)$ & 1.84 & 6.60 & 0.4 & 0.69 & 1.5 & $\gtrsim 26.5$ & $<0.40$ & $\mathrm{a}$ \\
\hline $2249+18(3 \mathrm{C} 454)$ & 1.76 & 2.10 & 11.9 & 0.72 & 5.0 & 0.8 & 4.29 & $\mathrm{~b}$ \\
\hline $2252+12(3 \mathrm{C} 455)$ & 0.54 & 13.70 & 1.7 & 0.62 & 0.2 & 348.2 & 0.06 & $\mathrm{a}$ \\
\hline $2342+82$ & 0.74 & 0.66 & $10-100$ & 0.79 & 4.5 & $\lesssim 1.3$ & 0.82 & $\mathrm{a}$ \\
\hline \multicolumn{9}{|l|}{ B3 VLA } \\
\hline $0809+404$ & 0.55 & 4.80 & 4.8 & 0.53 & 1.1 & 16.1 & 0.48 & $?$ \\
\hline $0810+460 \mathrm{~B}$ & 0.33 & 2.80 & 13.9 & 0.94 & 1.1 & 10.3 & 0.44 & $\mathrm{a}$ \\
\hline $1025+390 \mathrm{~B}$ & 0.361 & 5.10 & $>100$ & 0.65 & 0.8 & 6.0 & 1.40 & $a ?$ \\
\hline $1128+455$ & 0.40 & 1.90 & 1.8 & 0.53 & 1.5 & 17.3 & 0.18 & $?$ \\
\hline $1159+395$ & 2.37 & 0.35 & 2.8 & 0.38 & 4.7 & 1.6 & 0.35 & $?$ \\
\hline $1225+442$ & 0.22 & 0.50 & 2.5 & 0.67 & 0.8 & 40.3 & 0.02 & $?$ \\
\hline $1233+418$ & 0.25 & 2.70 & 3.5 & 0.51 & 0.6 & 52.7 & 0.08 & $\mathrm{a} ?$ \\
\hline $1242+410$ & 0.811 & 0.40 & 4.7 & 0.38 & 2.6 & 4.2 & 0.16 & $?$ \\
\hline $1350+432$ & 2.149 & 8.00 & 0.2 & 0.84 & 1.2 & 55.4 & 0.23 & $?$ \\
\hline
\end{tabular}




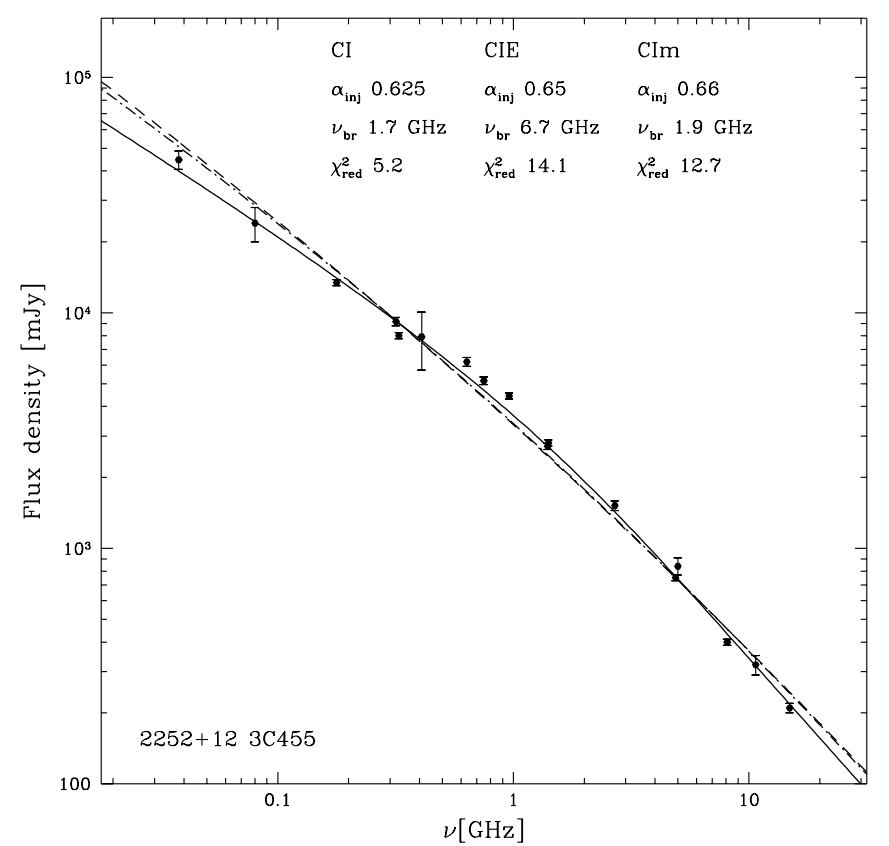

Fig. 3. Three different fit results for the source $2252+12$. The solid line corresponds to a pure CI model, the dashed line represents the CIE model, the dot-dashed line stands for the CIm model. Note the different qualities of the fits, expressed by the various $\chi_{\text {red }}^{2}$ values!

limit is set by confusion with the effects of absorption processes. The injection spectral index $\alpha_{\text {inj }}$ ranges from 0.35 to 0.8 , with $\left\langle\alpha_{\mathrm{inj}}>=0.63\right.$. The typical errors of the break frequencies and the injection spectral indices as given by the fit algorithm are up to $40 \%$ and 0.05 , respectively. The results of the CI fits are compiled in Tab. 2.

\subsection{Radiative ages and the nature of CSS sources}

In order to determine radiative ages, from Eq. (2) and variants of the other models, the magnetic field $B$ has to be known. We stress that the age depends rather strongly on $B$, which is somewhat uncertain. We take the equipartition value $B_{\text {eq }}$. Our assumption is motivated by the fact that $B_{\text {eq }}$ accounts rather well for the low-frequency turnover in terms of synchrotron self-absorption. We are aware that this is not a proof for equipartition. Other authors (e.g. Bicknell et al. 1997) prefer instead thermal absorption. In any case, $B_{\text {eq }}$ represents a poor statistical upper limit to $B$, in the sense that, were it larger by a factor of four, the low frequency turnovers would be systematically higher than observed by $\approx 30 \%$.

Using the value of $B_{\text {eq }}$, we obtain, from Eq. (2), radiative ages $\tau_{\text {syn }}$ ranging from $10^{3}$ to $10^{5}$ years. Since the intrinsic magnetic fields of the CSS sources in our sample strongly overweight the magnetic field equivalent
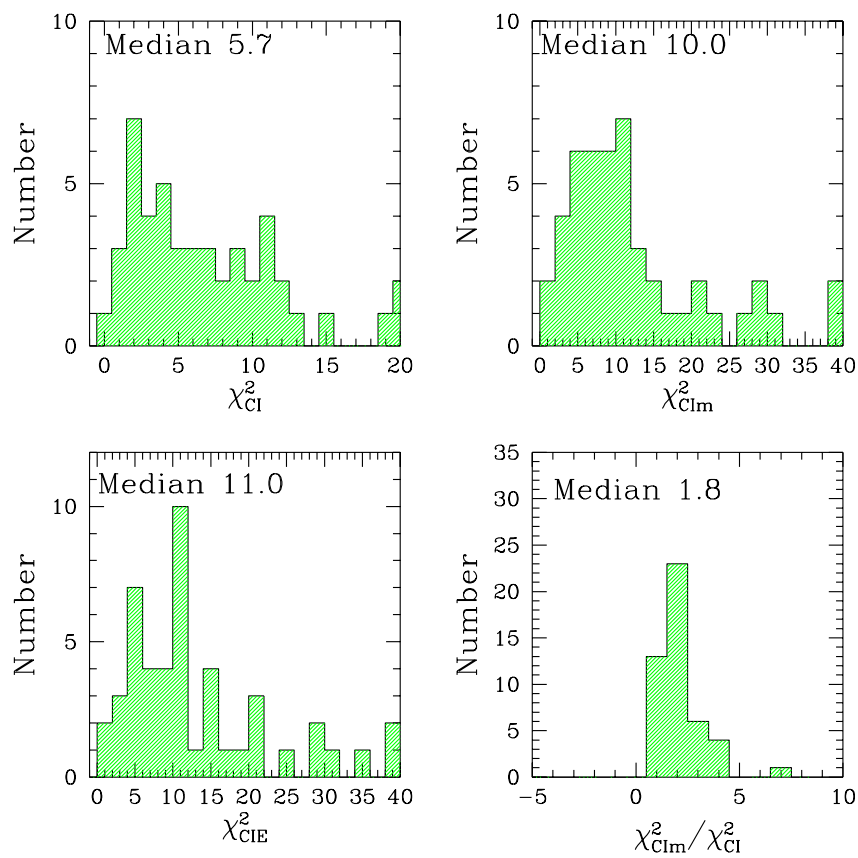

Fig. 4. $\chi_{\text {red }}^{2}$ histograms for the CI, CIE, and CIm models. On the bottom right the histogram of the ratio between the CIm and the CI $\chi_{\text {red }}^{2}$ is also shown.

to the cosmic microwave background $\left(B_{\mathrm{CMB}}\right)$, the latter can be neglected in Eq. (2). Therefore, if the source magnetic field deviates by a factor $f$ from the field determined for equipartition $B_{\text {eq }}$, the radiative ages will change by $f^{-3 / 2}$. These radiative ages do not necessarily represent the source ages, but rather the radiative ages of the dominant source component(s). Only when the lobes, which have accumulated the electrons produced over the source lifetime, dominate the source spectrum, the radiative age $\tau_{\text {syn }}$ is likely to represent the age of the source. If, instead, the spectrum is dominated by a jet or by hot spots, the radiative age likely represents the permanence time of the electrons in that component and is expected to be less (perhaps much less) than the source age. In addition, dominant jets or hot spots might have their break frequency up-shifted by Doppler effects.

The existing structure information on our sample, mostly from MERLIN and VLBI observations, allows us to split the sources in two groups: those in which the overall spectrum is dominated by lobes (classified "type a" in Tab. 2); those in which the spectrum is dominated by a bright jet or hot spot (classified "type b" in Tab. 2). For the B3 VLA sample the available information does not yet allow such a morphological sub-division.

The sources of class b have radiative ages systematically lower than those of class a, as we expected. Furthermore, while the synchrotron age is well correlated with the source size for class a, it seems that there is no corre- 


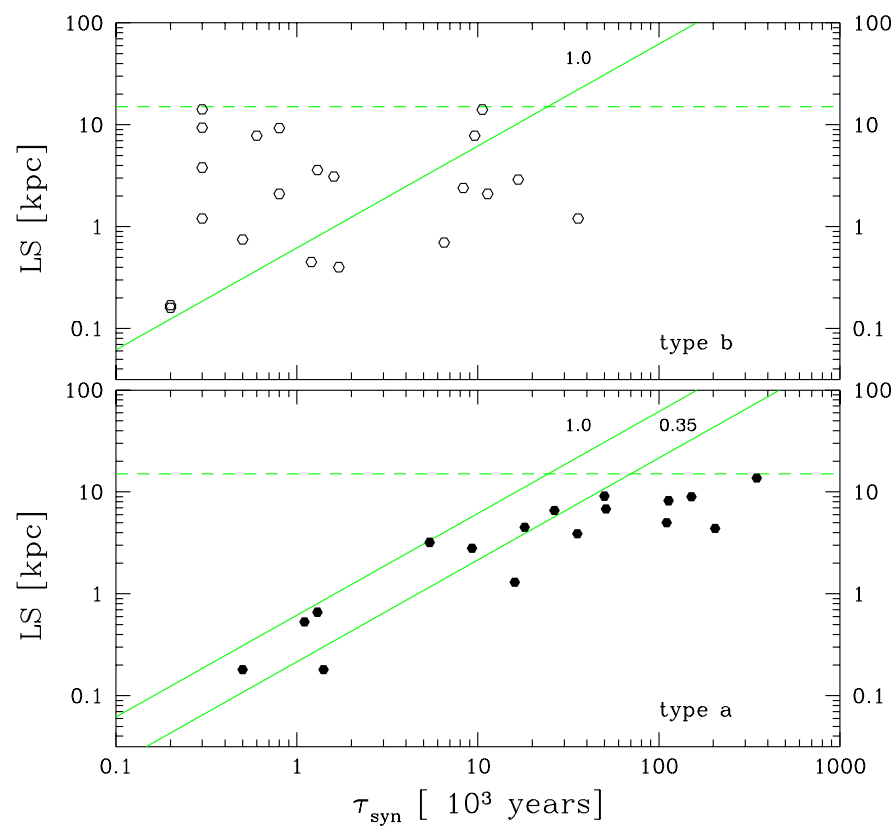

Fig. 5. Linear size as a function of the synchrotron age for type a (filled dots) and type b (open dots) sources. The B3 VLA sources have been excluded. The horizontal dashed lines represent the selection limits of the linear size distribution of the sources in our samples while the diagonal lines reflect constant values of $\frac{v_{\exp }}{c}$.

lation at all between the linear size and the radiative age for class b sources (see Fig. 5). We have further computed for each source the expansion velocity $v_{\exp } \approx \mathrm{LS} / 2 \tau_{\mathrm{syn}}$, where LS is the source largest dimension. The two classes show very different distributions:

Class a $\left\langle v_{\exp } / c>=0.34 \pm 0.07 \quad \sigma_{v_{\exp }}=0.28\right.$

Class $\mathrm{b}<v_{\exp } / c>=11 \pm 4.4 \quad \sigma_{v_{\exp }}=20$

Provided the assumed magnetic field is reasonably correct, the above values for the radiative ages indicate that the CSS sources are young. We further note that the ages, and corresponding expansion velocities, are not far (somewhat larger) from the recent results on expansion of CSOs by Owsianik et al. (1998) and Owsianik \& Conway (1998). As the radiative ages are strongly dependent on the assumed magnetic field, a field only a factor two lower than assumed would be required for a better agreement.

In order to maintain the frustration scenario, in which the sources' lifetimes are $\approx 10^{7}$ years, their equipartition magnetic field should be decreased by a factor $\gtrsim 20$.

One could think that the correlation between linear sizes and the radiative ages shown in Fig. 5 could be a partial consequence of the equipartition assumption. In fact, $B_{\text {eq }} \propto \mathrm{LS}^{-6 / 7}$ implies that LS $\propto \tau_{\mathrm{syn}}^{7 / 9}$. This is not the case for the following reasons: 1) no correlation between linear sizes and radiative ages is obvious for type b sources.

2) In particular, the break frequencies seem to be correlated with the linear sizes for type a sources (Fig. 6).

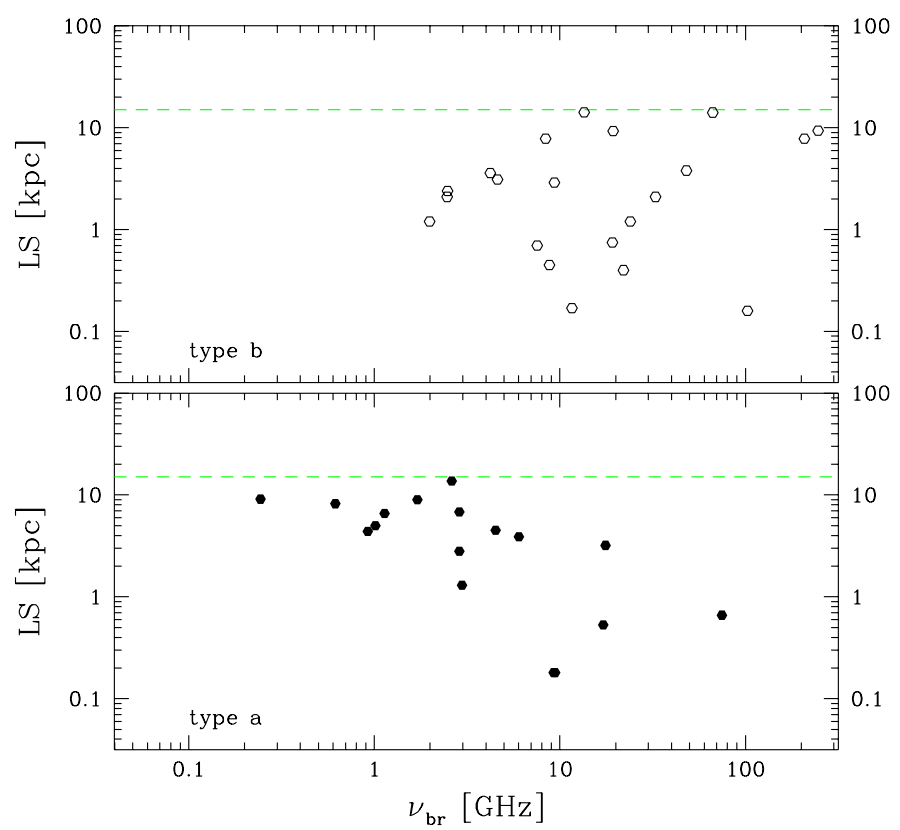

Fig. 6. Linear size as a function of the break frequency (in the source rest frame) for type a (filled dots) and type b (open dots) sources. The B3 VLA sources have been excluded. The horizontal dashed lines represent the selection limits of the linear size distribution of the sources in our samples.

These means, at least for class a sources, that the break frequency is an effective clock indicating the source age. The correlation seen in Fig. 5 is not an artifact completely introduced by the assumption of equipartition in Eq. (2).

\section{Conclusions}

The high-frequency integrated spectrum of CSS sources shows a break with a moderate spectral steepening $(\Delta \alpha \approx$ 0.5 ), well fitted by the continuous injection model spectrum with constant magnetic field. Spectral fits with synchrotron models with decreasing magnetic field are definitely poorer. There is no evidence of sharp cut-offs as would be expected if the supply of relativistic electrons had stopped more than $\approx 10^{4}$ years ago.

In lobe dominated CSS sources the radiative ages $\tau_{\text {syn }}$ are likely to represent the source ages. Assuming equipartition magnetic field strengths the source ages are in the range of up to $10^{5}$ years, in agreement with the recent results on expansion velocities of small size CSOs by Owsianik et al. (1998) and Owsianik \& Conway (1998) derived from VLBI observations. Therefore, assuming the CSS sources to be young, the magnetic field has been deduced to be equal to the equipartition value within a factor $\approx 2$.

In jet or hot spot dominated CSS sources, the radiative life times are much shorter and very likely represent the permanence time of the electrons in those components. 
Acknowledgements. We are grateful to Drs. B. Cotton and S. Spangler for very helpful comments on the manuscript of this paper and to Dr. H. Andernach for the suggestions on the compilation of the flux densities. We made use of the database CATS (Verkhodanov et al. 1997) of the Special Astrophysical Observatory. We acknowledge the Italian Ministry for University and Scientific Research (MURST) for partial financial support (grant Cofin98-02-32). Part of this work was supported by European Commission, TMR Programme, Research Network Contract ERBFMRXCT97-0034 "CERES".

\section{Appendix}

\section{A. Synchrotron radiation, adiabatic expansion and continuous injection in the case of a time- variable magnetic field}

We assume that adiabatic expansion and injection of particles start at time $t_{0}$. The source is continuously replenished by a constant flow of particles with a power law energy spectrum with spectral index $\delta$ intensity $B$ decreases according to

$$
B=B_{0}\left(\frac{t_{0}}{t}\right)^{m}
$$

and the characteristic size $R$ of the regions occupied by the particles (which should not be confused with the source size LS) grows as

$$
R=R_{0}\left(\frac{t}{t_{0}}\right)^{r}
$$

The continuity equation for this model is

$$
\frac{\partial N}{\partial t}=a_{1} \frac{\partial}{\partial \epsilon}\left(\epsilon^{2} N\right)+a_{2} \frac{\partial}{\partial \epsilon}(\epsilon N)+a_{3} \epsilon^{-\delta}
$$

$N(\epsilon, t)$ is the differential energy spectrum of the relativistic particles at time t. The first term on the right hand side describes the effects of the synchrotron losses, $a_{1}=c_{1} B_{\perp}^{2}$, $c_{1}$ is constant (Pacholczyk 1970). The second term accounts for adiabatic expansion $\left(a_{2}=r / t\right)$. The term $a_{3}$ describes the injection rate and is considered to be constant for simplicity.

The solution of Eq. (A1) is

$$
\begin{aligned}
N(\epsilon, t) & =a_{3} \epsilon^{-\delta} \cdot \\
& \int_{t_{\text {low }}}^{t}\left(\frac{\tau^{\prime}}{t}\right)^{r(\delta-1)}\left(1-\frac{\epsilon}{\epsilon_{\mathrm{br}}^{*}\left(\tau^{\prime}\right)}\right)^{\delta-2} d \tau^{\prime}
\end{aligned}
$$

where $t_{\text {low }}$ is $\operatorname{MAX}\left[t_{0}, \tau_{\text {min }}\right]$ and $\tau_{\text {min }}$ is implicitly given by

$$
\epsilon=\frac{2 m+r-1}{c_{1} B_{\perp}^{2} t\left[\left(\frac{t}{\tau_{\min }}\right)^{2 m+r-1}-1\right]}
$$

The energy $\epsilon_{\mathrm{br}}^{*}(\tau)$ is

$$
\epsilon_{\mathrm{br}}^{*}(\tau)=\frac{2 m+r-1}{c_{1} B_{\perp}^{2} t\left((t / \tau)^{2 m+r-1}-1\right)}
$$

$\epsilon_{\mathrm{br}}^{*}(\tau)$ is the break energy of the particle population injected at the time $\tau\left(t_{0} \leq \tau \leq t\right)$. The solution (A2) represents the sum of all the populations injected form $\tau=t_{0}$ to $\tau=t$. The integral (A2) can be written as

$$
\begin{aligned}
N(\epsilon, t) & =a_{3} \epsilon^{-\delta-1} t \frac{\epsilon_{\mathrm{br}}}{2 m+r-1} . \\
& \int_{z_{\mathrm{low}}}^{1}\left[(1-z) \frac{\epsilon_{\mathrm{br}}}{\epsilon}+1\right]^{-\frac{r \delta+2 m}{2 m+r-1}} z^{\delta-2} d z
\end{aligned}
$$


where $z_{\text {low }}$ is equal to $\operatorname{MAX}\left[0,1-\epsilon / \epsilon_{\text {brmin }}\right]$,

$\epsilon_{\mathrm{br}}=\frac{2 m+r-1}{c_{1} B_{\perp}^{2} t}$

and

$\epsilon_{\mathrm{brmin}}=\frac{\epsilon_{\mathrm{br}}}{\left(t / t_{0}\right)^{2 m+r-1}-1}$

In the energy spectrum two break energies are always present, $\epsilon_{\mathrm{br}}$ and $\epsilon_{\mathrm{brmin}}$, the break energy of the first population injected at $t=t_{0}$. These regions can be identified in the energy spectrum:

1) $\epsilon \ll \epsilon_{\text {brmin }}$.

In this region the spectrum is a power law:

$N(\epsilon, t) \simeq \frac{a_{3} \epsilon^{-\delta} t}{r(\delta-1)+1}\left[1-\left(\frac{t_{0}}{t}\right)^{r(\delta-1)+1}\right]$

2) $\epsilon_{\mathrm{brmin}} \approx \epsilon<\epsilon_{\mathrm{br}}$.

The spectrum starts to gradually deviate from the low energy power law at $\epsilon \approx \epsilon_{\mathrm{brmin}}$ and undergoes a steepening at $\epsilon \approx \epsilon_{\mathrm{br}}$.

$$
\begin{aligned}
N(\epsilon, t)= & a_{3} \epsilon^{-\delta-1} t \frac{\epsilon_{\mathrm{br}}}{2 m+r-1} . \\
& \int_{z_{\text {low }}}^{1}\left[(1-z) \frac{\epsilon_{\mathrm{br}}}{\epsilon}+1\right]^{-\frac{r \delta+2 m}{2 m+r-1}} z^{\delta-2} d z
\end{aligned}
$$

3) $\epsilon \gg \epsilon_{\mathrm{br}}$.

The spectrum is again a power law but with a spectral index steeper by 1 .

$$
N(\epsilon, t) \simeq \frac{a_{3} \epsilon^{-\delta-1}}{c_{1} B_{\perp}^{2}(\delta-1)}
$$

Since the separation between these two energies increases with time the shape of the spectrum also changes with time. But an asymptotic stationary shape still exists when $t / t_{0} \rightarrow \infty$. In fact, in this case $\epsilon_{\mathrm{brmin}} \rightarrow 0$, and at every energy $\epsilon, z_{\text {low }}=0$. In this way the integral

$$
\begin{aligned}
N(\epsilon, t)= & a_{3} \epsilon^{-\delta-1} t \frac{\epsilon_{\mathrm{br}}}{2 m+r-1} . \\
& \int_{0}^{1}\left[(1-z) \frac{\epsilon_{\mathrm{br}}}{\epsilon}+1\right]^{-\frac{r \delta+2 m}{2 m+r-1}} z^{\delta-2} d z
\end{aligned}
$$

does not depend on the ratio $t / t_{0}$ anymore.

The integral can be solved only by numerical means, but analytic asymptotic limits can still be found.

$$
\begin{aligned}
N(\epsilon, t) & \simeq \frac{a_{3} \epsilon^{-\delta} t}{r(\delta-1)+1} & & \epsilon \ll \frac{2 m+r-1}{c_{1} B_{\perp}^{2} t} \\
N(\epsilon, t) & \simeq \frac{a_{3} \epsilon^{-\delta-1}}{c_{1} B_{\perp}^{2}(\delta-1)} & & \epsilon \gg \frac{2 m+r-1}{c_{1} B_{\perp}^{2} t}
\end{aligned}
$$

i.e. in the spectrum only the $\epsilon_{\text {br }}$ break energy is present. The asymptotic solutions are two power laws. The lowenergy power law spectral index is $\delta$, the high-energy power law spectral index is $\delta+1$. Moreover, the normalization for $\epsilon \gg \epsilon_{\mathrm{br}}$ is time-independent, i.e. a perfect balance is reached between the number of particles that leave this region because the synchrotron and expansion losses and the number of particles constantly injected into the source.

\section{A.1. CI model}

The CI model reproduces the simple situation in which there is no adiabatic expansion and the magnetic field strength stays constant. This corresponds to $r=0$ and $m=0$ in (A3). In this case the integral can be directly solved resulting in:

$$
\begin{array}{lll}
N(\epsilon, t)=\frac{a_{3} \epsilon^{-\delta-1}}{c_{1} B_{\perp}^{2}(\delta-1)}\left[1-\left(1-\epsilon / \epsilon_{\mathrm{br}}\right)^{\delta-1}\right] & & \epsilon<\epsilon_{\mathrm{br}} \\
N(\epsilon, t)=\frac{a_{3} \epsilon^{-\delta-1}}{c_{1} B_{\perp}^{2}(\delta-1)} & \epsilon \geq \epsilon_{\mathrm{br}}
\end{array}
$$

where

$\epsilon_{\mathrm{br}}=\frac{1}{c_{1} B_{\perp}^{2}\left(t-t_{0}\right)}$

The shape of the spectrum does not depend on time (Kardashev 1962).

\section{A.2. CIE model}

The CIE model reproduces the situation in which the volume containing the particles is adiabaticlly expanding at a constant rate and the magnetic field is frozen in the plasma. Since $R \propto t$, the conservation of the magnetic flux requires that $B \propto t^{-2}$. This corresponds to $r=1$ and $m=2$ in (A3). The shape of the spectrum changes with time during its rise. However an asymptotic stationary solution exists when $t \gg t_{0}$ :

$$
\begin{aligned}
& N(\epsilon, t) \simeq \frac{a_{3} \epsilon^{-\delta-1} t}{c_{1} B_{\perp}^{2}} \int_{0}^{1}\left[(1-z) \frac{\epsilon_{\mathrm{br}}}{\epsilon}+1\right]^{-\frac{\delta}{4}-1} z^{\delta-2} d z \\
& \epsilon_{\mathrm{br}}=\frac{4}{c_{1} B_{\perp}^{2} t}
\end{aligned}
$$

In this asymptotic limit the CIE spectrum shows a break energy four time greater than the break energy of the CI model. Using (A4) one finds that

$$
\begin{array}{lll}
N(\epsilon, t) \simeq \frac{a_{3} \epsilon^{-\delta} t}{\delta} & & \epsilon \ll \frac{4}{c_{1} B_{\perp}^{2} t} \\
N(\epsilon, t) \simeq \frac{a_{3} \epsilon^{-\delta-1}}{c_{1} B_{\perp}^{2}(\delta-1)} & \epsilon \gg \frac{4}{c_{1} B_{\perp}^{2} t}
\end{array}
$$

this solution is the same found by Kardashev (1962). The asymptotic solutions are two power laws with the same dependence on the spectral index as in the case of the CI model. However, the energy range necessary to complete 
the transition between the two asymptotic power laws is wider. For $t \gg t_{0}$ the break energy of the CIE model is greater than the break energy $\epsilon_{\text {brmin }}$ of a CI model with the same final magnetic field. This result appears a bit surprising at first glance. In fact, due to the expansion losses and the stronger magnetic field, the break energy of the first population of particle injected at $t_{0}$ in the CIE model is lower than that of the corresponding population of the CI model. However, both the stronger mean magnetic field and the expansion losses will also decrease the energy of the first populations in a way that, at time $t$, they do not contribute appreciably to the integrated spectra.

\section{A.3. CIm model}

The CIm model reproduces the situation in which the volume containing the particles is adiabaticlly expanding and there is always equipartition between magnetic field and particle energy density. The equipartition condition $u \propto B^{2}$ ( $u$ is the energy density of the relativistic particles) implies that $R \propto t^{-m / 2}$. This corresponds to $r=-m / 2$ in (A3). An asymptotic stationary solution exists when $t \gg t_{0}$ :

$N(\epsilon, t) \simeq \frac{a_{3} \epsilon^{-\delta-1} t}{c_{1} B_{\perp}^{2}} \int_{0}^{1}\left[(1-z) \frac{\epsilon_{\mathrm{br}}}{\epsilon}+1\right]^{-\frac{m \delta+4 m}{5 m-2}} z^{\delta-2} d z$

$\epsilon_{\mathrm{br}}=\frac{2.5 m-1}{c_{1} B_{\perp}^{2} t}$

In this asymptotic limit the CIm spectrum shows a break energy $(2.5 m-1)$ time greater than the break energy of the CI model. Using (A4) one finds that

$\begin{array}{lll}N(\epsilon, t) \simeq \frac{a_{3} \epsilon^{-\delta} t}{\frac{m}{2}(\delta-1)+1} & & \epsilon \ll \frac{2.5 m-1}{c_{1} B_{\perp}^{2} t} \\ N(\epsilon, t) \simeq \frac{a_{3} \epsilon^{-\delta-1}}{c_{1} B_{\perp}^{2}(\delta-1)} & \epsilon \gg \frac{2.5 m-1}{c_{1} B_{\perp}^{2} t}\end{array}$

For the CIm the CIE comments are also valid.

\section{A.4. Emission spectrum}

The emission spectrum is given by the convolution of the emission spectrum of the single electron $F\left(\nu /\left(c_{2} B \sin \theta \epsilon^{2}\right)\right)$ (with $c_{2}$ constant, Pacholczyk 1970) with the energy distribution $N(\epsilon, t)$ :

$$
\begin{aligned}
\mathrm{S}_{\text {aged }}\left(\nu / \nu_{\mathrm{br}}, \alpha_{\mathrm{inj}}\right) \propto & \int_{0}^{\frac{\pi}{2}} \int_{0}^{\infty} F\left(\frac{\nu / \nu_{\mathrm{br}}}{x^{2} \sin \theta}\right) \\
& N\left(x \cdot \epsilon_{\mathrm{br}}, t\right) \sin ^{2} \theta d x d \theta
\end{aligned}
$$

where $\nu_{\mathrm{br}}=c_{2} B \epsilon_{\mathrm{br}}{ }^{2}$ and $\alpha_{\mathrm{inj}}=(\delta-1) / 2$. It is assumed that the pitch angles $\theta$ between the electron velocity and the magnetic field direction are isotropically distributed and that the time scale for their continuous re-isotropization is much shorter than the radiative timescale, $c_{1}\left\langle B_{\perp}^{2}\right\rangle=\frac{2}{3} c_{1} B^{2}$ (Jaffe \& Perola 1974).

\section{References}

Altenhoff W.J., Baars J.W.M., Downes D., Wink J.E., 1987, A\&A 184, 381

Baars J.W.M., Genzel R., Pauliny-Toth I.I.K., Witzel A., 1977, A\&AS 61, 99

Begelman M.C.I., 1996, in Cygnus A - Study of a Radio Galaxy, ed. C.L. Carilli \& D.E. Harris (Cambridge University Press) p. 209

Baldwin J. 1982, in Extragalactic Radio Sources ed. D.S. Heeschen \& C.M. Wade (Reidel) p. 21

Bicknell G.V., Dopita M.A., O’Dea C.P.O., 1997, ApJ 485, 112

van Breugel W.J.M., Miley G.K., Heckman T.A., 1984, AJ 89, 5

Carvahlo J.C., 1985, MNRAS 215, 463

Fanti R., Fanti C., Schilizzi R.T. et al., 1990, A\&A 231, 333

Fanti C., Fanti R., Dallacasa D. et al., 1995, A\&A 302, 317

Jaffe W.J., Perola G.C., 1974, A\&A 26, 423

Kardashev N.S., 1962, SvA 6, 317

Kreysa E., Gemünd H.-P., Gromke J. et al., 1998, Proc. of the SPIE "Astronomical Telescopes and Instrumentation", 3357,319

Kühr H., Witzel A., Pauliny-Toth I.I.K., Nauber U., 1979, MPIfR Preprint No. 55

Owsianik I., Conway J.E., Polatidis A.G., 1998, A\&A 336, L37

Owsianik I, Conway J.E., 1998, A\&A 337, 69

Pacholczyk A.G., 1970, 'Radio Astrophysics', Freeman \& Co., San Francisco

Peacock J.A., Wall J.V., 1981, MNRAS 194, 19

Phillips T.J., Mutel R.L., 1982, A\&A 106, 21

Verkhodanov O.V., Trushkin S.A., Andernach H., Chernenkov V.N., 1997 in Astronomical Data Analysis Software and Systems VI ed. Gareth Hunt and H. E. Payne (ASP Conference Series) 125, 322

Vigotti M., Grueff G., Perley R., Clark B.G., Bridle A.J., 1989 AJ 98,419

This article was processed by the author using Springer-Verlag $\mathrm{LAT}_{\mathrm{E}} \mathrm{X}$ A\&A style file $L-A A$ version 3 . 